\title{
Existence of the weak solutions for the compressible magnetohydrodynamic flows driven by stochastic forcing
}

Junling Sun ${ }^{1 *}$ and Hengyan $\mathrm{Li}^{2}$

\section{"Correspondence:}

junlingsunmath121@163.com ${ }^{1}$ School of Mathematics and Information Science, Henan Polytechnic University, Jiaozuo, 454150, P.R. China

Full list of author information is available at the end of the article

\begin{abstract}
In this paper, we study the existence of weak solutions for the compressible magnetohydrodynamics flows driven by stochastic external forcing. Our method is based on solving the system for each fixed representative of the random variable and applying an abstract result on the measurability of multi-valued maps.
\end{abstract}

Keywords: stochastic magnetohydrodynamics; compressible fluid; random driving force

\section{Introduction and main results}

This paper considers the following compressible magnetohydrodynamic flows driven by a stochastic external force in the isentropic case [1-3]:

$$
\begin{aligned}
& d \rho+\operatorname{div}_{x}(\rho \mathbf{u}) d t=0, \\
& d(\rho \mathbf{u})+\left(\operatorname{div}_{x}(\rho \mathbf{u} \otimes \mathbf{u})+\nabla P(\rho)-(\nabla \times \mathbf{H}) \times \mathbf{H}\right. \\
& \left.\quad-v \Delta \mathbf{u}-(v+\lambda) \nabla\left(\mathbf{d i v}_{x} \mathbf{u}\right)\right) d t=\rho d W, \\
& d \mathbf{H}-\left(\nabla \times(\mathbf{u} \times \mathbf{H})+\nabla \times\left(v^{\prime} \nabla \times \mathbf{H}\right)\right) d t=0,
\end{aligned}
$$

where $\rho(t, x, \omega), \mathbf{u}(t, x, \omega), \mathbf{H}(t, x, \omega)$ are functions of the time $t \in(0, T)$, the spatial position $x \in \Omega$, and $\omega \in \mathcal{O}=\{\mathcal{O}, \mathcal{B}, \mu\}, \mathcal{O}$ is a topological probability case, with the family of Borel sets $\mathcal{B}$, and a regular probability measure $\mu$. The symbol $\otimes$ denotes the Kronecker tensor product. $\mathbf{H}(t, x, \omega)$ satisfies

$$
\operatorname{div}_{x} \mathbf{H}=0 \text {, }
$$

$P(\rho)$ is the pressure, the viscosity coefficients of the flow satisfy $2 v+3 \lambda>0$ and $v>0$; $v^{\prime}>0$ is the magnetic diffusivity acting as a magnetic diffusion coefficient of the magnetic field, and all these kinetic coefficients and the magnetic diffusivity are independent of the magnitude and direction of the magnetic field, the perturbation $W$ is a random variable represented for a.a. $\omega$ by a bounded function, sufficiently regular with respect to the spatial variable $x \in \Omega$. Moreover, we supplement this with the no-slip boundary con-

(c) 2015 Sun and Li; licensee Springer. This article is distributed under the terms of the Creative Commons Attribution 4.0 International License (http://creativecommons.org/licenses/by/4.0/), which permits unrestricted use, distribution, and reproduction in any medium, provided you give appropriate credit to the original author(s) and the source, provide a link to the Creative Commons license, and indicate if changes were made. 
dition,

$$
\left.\mathbf{u}\right|_{\partial \Omega}=0,\left.\quad \mathbf{H}\right|_{\partial \Omega}=0,
$$

and the initial conditions

$$
\begin{aligned}
& \rho(0, \cdot)=\rho_{0}, \quad \rho \mathbf{u}(0, \cdot)=(\rho \mathbf{u})_{0}, \quad(\rho \mathbf{u})_{0}=0, \quad \text { if } \rho_{0}=0, \\
& \mathbf{H}(0, \cdot)=\mathbf{H}_{0}, \quad \operatorname{div}_{x} \mathbf{H}_{0}=0 .
\end{aligned}
$$

Although the electric field does not appear in the MHD equations (1.1)-(1.3), from (1.3), the electric field $\mathbf{E}$ is induced by moving conductive flow in the magnetic field, having the following relationship with the magnetic field $\mathbf{H}$ and the velocity $\mathbf{u}$ :

$$
\mathbf{E}=v^{\prime} \nabla \times \mathbf{H}-\mathbf{u} \times \mathbf{H}
$$

The stochastic incompressible and compressible fluid systems have attracted great attention by considering that a system in reality is usually affected by external perturbations which in many cases are of great uncertainty or random influence. These random effects are introduced not only to compensate for the defects in some deterministic models but also to reveal the intrinsic phenomena. In this direction, the surveys corresponding to the stochastic case are in [4-6], and much work has been done on the so-called weak solutions in the probabilistic sense (or martingale solutions) for the stochastic incompressible Navier-Stokes equations; for example, see [7-9]. For the one dimensional compressible stochastic Navier-Stokes system, we refer to $[10,11]$ for details. Most recently, Feireisl et al. [12] gave results on the existence of weak solutions for the three dimensional stochastic compressible Navier-Stokes system. Their results included a number of stochastic external forces, e.g. Lévy noise.

The main difficulty of the study of MHD driven by stochastic external forcing is the presence of the magnetic field and its interaction with the hydrodynamic motion in the MHD flow of large oscillation. Inspired by the work of [12], this paper is devoted to the study of the existence of weak solutions for three dimensional stochastic compressible magnetohydrodynamic flows. One of the difficulties is to obtain a suitable energy estimate when there is a magnetic field and its interaction with the hydrodynamic motion is driven by the stochastic external forcing. To the best of our knowledge, there are only results on the existence of weak solutions for the stochastic incompressible magnetohydrodynamic flows, $c f .[13,14]$.

Inspired by the definition of the weak solutions to the compressible Naiver-Stokes equation in [12], we give the definition of the weak solutions to the problem (1.1)-(1.4).

The random variables $\rho(t, x, \omega), \mathbf{u}(t, x, \omega), \mathbf{H}(t, x, \omega)$ is said to be a weak solution to the problem (1.1)-(1.4) of the compressible MHD equations with stochastic external forces if the following conditions hold:

- The density $\rho \geq 0$ :

$$
\begin{aligned}
& \rho(\cdot, \omega) \in \mathbf{C}\left([0, T] ; \mathbf{L}^{1}(\Omega)\right), \\
& \rho \in \mathbf{L}^{\infty}\left(0, T: \mathbf{L}^{q}\right) \text { for certain } q>1 \text { for a.a. } \omega \in \mathcal{O},
\end{aligned}
$$


and the family of integral identities

$$
\begin{aligned}
& \int_{\Omega}(\rho(\tau, x, \omega)+b(\rho(\tau, x, \omega))) \varphi(x) d x-\int_{\Omega}\left(\rho_{0}(x, \omega)+b\left(\rho_{0}(x, \omega)\right)\right) \varphi(x) d x \\
&= \int_{0}^{\tau} \int_{\Omega}\left(\rho(t, x, \omega) \mathbf{u}(t, x, \omega) \cdot \nabla_{x} \varphi(x)\right. \\
&\left.\quad+\left(b(\rho(t, x, \omega))-b^{\prime}(\rho(t, x, \omega)) \rho(t, x, \omega) \operatorname{div}_{x} \mathbf{u}(t, x, \omega) \varphi(x)\right)\right) d x d t
\end{aligned}
$$

for any test function $\varphi \in \mathbf{C}^{1}(\bar{\Omega})$, any $b \in \mathbf{C}_{c}^{\infty}[0, \infty)$, and a.a. $\omega \in \mathcal{O}$.

- The momentum equation:

$$
\begin{aligned}
& \mathbf{u}(\cdot, \omega) \in \mathbf{L}^{2}\left(0, T ; \mathbf{W}_{0}^{1,2}\left(\Omega ; \mathbf{R}^{3}\right)\right) \quad \text { for a.a. } \omega \in \mathcal{O}, \\
& \rho \mathbf{u} \in \mathbf{L}^{\infty}\left(0, T: \mathbf{L}^{\frac{2 q}{q+1}}\left(\Omega ; \mathbf{R}^{3}\right)\right), \\
& \rho(\mathbf{u}-W) \in \mathbf{C}_{\text {weak }}\left([0, T] ; \mathbf{L}^{\frac{2 q}{q+1}}\left(\Omega ; \mathbf{R}^{3}\right)\right) \quad \text { for a.a. } \omega \in \Omega, \\
& P(\rho) \in \mathbf{L}^{\infty}\left(0, T: \mathbf{L}^{1}(\Omega)\right) \cap \mathbf{L}^{\gamma}((0, T) \times \Omega) \quad \text { for some } \gamma>1 \text { for a.a. } \omega \in \mathcal{O},
\end{aligned}
$$

and the following weak formulations hold:

$$
\begin{aligned}
\int_{\Omega}( & (\tau, x, \omega)(\mathbf{u}(\tau, x, \omega)-W(\tau, x, \omega)) \cdot \varphi(x)) d x-\int_{\Omega}(\rho \mathbf{u})_{0}(x, \omega) \cdot \varphi(x) \\
= & \int_{0}^{\tau} \int_{\Omega}\left(\rho(t, x, \omega) \mathbf{u}(t, x, \omega) \otimes \mathbf{u}(t, x, \omega): \nabla_{x} \varphi(x)+P(\rho(t, x, \omega)) \mathbf{d i v}_{x} \varphi(x)\right) d x d t \\
& -\int_{0}^{\tau} \int_{\Omega}\left(\frac{1}{2}|\mathbf{H}(t, x, \omega)|^{2} \cdot \nabla_{x} \varphi(x)+(\mathbf{H}(t, x, \omega) \cdot \nabla) \mathbf{H}(t, x, \omega) \cdot \varphi(x)\right) d x d t \\
& -\int_{0}^{\tau} \int_{\Omega}\left(v \nabla_{x} \mathbf{u}(t, x, \omega) \cdot \nabla_{x} \varphi(x)+(v+\lambda)\left(\operatorname{div}_{x} \mathbf{u}(t, x, \omega)\right) \cdot \nabla_{x} \varphi(x)\right. \\
& \left.+\rho(t, x, \omega) \mathbf{u}(t, x, \omega) \cdot \nabla_{x}(W(t, x, \omega) \varphi(x))\right) d x d t
\end{aligned}
$$

for all $\varphi \in \mathbf{C}_{c}^{\infty}\left(\Omega ; \mathbf{R}^{3}\right)$, and for a.a. $\omega \in \mathcal{O}$.

- The induction equation of electromagnetism:

$$
\mathbf{H}(\cdot, \omega) \in \mathbf{L}^{2}\left(0, T ; \mathbf{W}_{0}^{1,2}\left(\Omega ; \mathbf{R}^{3}\right)\right) \quad \text { for a.a. } \omega \in \mathcal{O},
$$

and the following weak formulations hold:

$$
\begin{gathered}
\int_{0}^{T} \int_{\Omega}\left((d \mathbf{H}(\tau, x, \omega), \varphi(x))+v^{\prime}\left(\nabla_{x} \times \mathbf{H}(\tau, x, \omega), \nabla_{x} \times \varphi(x)\right)\right. \\
\left.-\left(\mathbf{u}(\tau, x, \omega) \times \mathbf{H}(\tau, x, \omega), \nabla_{x} \times \varphi(x)\right)\right) d x d t=0
\end{gathered}
$$

for all $\varphi \in \mathbf{C}_{c}^{\infty}\left(\Omega ; \mathbf{R}^{3}\right)$, and for a.a. $\omega \in \mathcal{O}$.

- The energy inequality:

$$
\begin{gathered}
\int_{\Omega}\left(\frac{1}{2} \rho(\tau, x, \omega)|\mathbf{u}(\tau, x, \omega)-W(\tau, x, \omega)|^{2}+P(\rho(\tau, x, \omega))+\frac{1}{2}|\mathbf{H}(\tau, x, \omega)|^{2}\right) d x \\
+\int_{\Omega}\left(v\left|\nabla_{x} \mathbf{u}(t, x, \omega)\right|^{2}+(v+\lambda)\left|\operatorname{div}_{x} \mathbf{u}(t, x, \omega)\right|^{2}+v^{\prime}\left|\nabla_{x} \times \mathbf{H}\right|^{2}\right) d x
\end{gathered}
$$




$$
\begin{aligned}
\leq & \int_{\Omega}\left(\frac{1}{2} \rho(s, x, \omega)|\mathbf{u}(s, x, \omega)-W(s, x, \omega)|^{2}+P(\rho(s, x, \omega))+\frac{1}{2}|\mathbf{H}(s, x, \omega)|^{2}\right) d x \\
& -\int_{0}^{\tau} \int_{\Omega}\left(\rho \mathbf{u} \otimes \mathbf{u}: \nabla_{x} W+P(\rho) \mathbf{d i v}_{x} W-\frac{1}{2} \rho \mathbf{u} \cdot \nabla_{x}|W|^{2}\right)(t, x, \omega) d x d t \\
& -\int_{0}^{\tau} \int_{\Omega}\left(v \nabla_{x} \mathbf{u} \cdot \nabla_{x} W+(v+\lambda) \mathbf{d i v}_{x} \mathbf{u} \cdot \nabla_{x} W\right)(t, x, \omega) d x d t \\
& +\int_{0}^{\tau} \int_{\Omega}\left(\frac{1}{2}|\mathbf{H}|^{2} \cdot \nabla_{x} W+(\mathbf{H} \cdot \nabla) \mathbf{H} \cdot W\right)(t, x, \omega) d x d t \\
& +\int_{0}^{\tau} \int_{\Omega}\left(\mathbf{u} \times \mathbf{H}, \nabla_{x} \times W\right)(\tau, x, \omega) d x d t
\end{aligned}
$$

for a.a. $\tau \geq s \geq 0$ including $s=0$, and a.a. $\omega \in \mathcal{O}$, with

$$
\mathcal{P}(\rho)=\rho \int_{0}^{\rho} \frac{P(z)}{z^{2}} d z
$$

We remark that the family of integral identities (1.5) is taken from [12], which is a weak formulation of the renormalized equation of continuity introduced by [15]. In order to derive the family of integral identities (1.6)-(1.7), we use the following well-known identities:

$$
\begin{aligned}
& d(\rho \mathbf{u})-\rho d W=d[\rho(\mathbf{u}-W)]-\operatorname{div}_{x}(\rho \mathbf{u}) W d t, \\
& (\nabla \times \mathbf{H}) \times \mathbf{H}=\frac{1}{2} \nabla\left(|\mathbf{H}|^{2}\right)-(\mathbf{H} \cdot \nabla) \mathbf{H}, \\
& \nabla \times(\nabla \times \mathbf{H})=\nabla \operatorname{div} \mathbf{H}-\Delta \mathbf{H}, \\
& \nabla \times(\mathbf{u} \times \mathbf{H})=(\operatorname{div} \mathbf{H}+\mathbf{H} \cdot \nabla) \mathbf{u}-(\operatorname{div} \mathbf{u}+\mathbf{u} \cdot \nabla) \mathbf{H} .
\end{aligned}
$$

Before stating our main result, we introduce the function space $\mathcal{I D}$ for the data as

$$
\begin{aligned}
\mathcal{I D}= & \left\{\left[\rho_{0},(\rho \mathbf{u})_{0}, \mathbf{H}_{0}\right] \mid \rho_{0} \in \mathbf{L}^{q}(\Omega),\left(\rho \mathbf{u}_{0}\right) \in \mathbf{L}^{\frac{2 q}{q+1}}\left(\Omega ; \mathbf{R}^{3}\right), \mathbf{H}_{0} \in \mathbf{L}^{2}(\Omega), \rho_{0} \geq 0,\right. \\
& \left.\int_{\Omega} \rho_{0} d x=M>0, \int_{\Omega} \frac{1}{2} \frac{\left|(\rho \mathbf{u})_{0}\right|^{2}}{\rho_{0}}+P\left(\rho_{0}\right)+\frac{1}{2}\left|\mathbf{H}_{0}\right|^{2} d x \leq E_{0}\right\},
\end{aligned}
$$

which is a closed convex subset of the separable Banach space $\mathbf{L}^{q}(\Omega) \times \mathbf{L}^{\frac{2 q}{q+1}}\left(\Omega ; \mathbf{R}^{3}\right) \times$ $\mathbf{W}_{0}^{1,2}(\Omega)$. In particular, it is a Suslin space.

Theorem 1.1 Let $\Omega \in \mathbf{R}^{3}$ be a bounded Lipschitz domain. Assume that the pressure $P \in$ $\mathbf{C}[0, \infty) \cap \mathbf{C}^{2}[0, \infty)$ and satisfies

$$
P(0)=0, \quad P^{\prime}(0)>0, \quad \lim _{\rho \rightarrow \infty} \frac{P^{\prime}(\rho)}{\rho^{p-1}}=P_{\infty}>0, \quad p>\frac{3}{2},
$$

and $\mathcal{W}$ is a complete separable metric space satisfying:

(i)

$$
\mathcal{W} \subset \mathbf{L}_{\text {weak }}^{\infty}\left(0, T ; \mathbf{W}_{0}^{1, \infty}\left(\Omega ; \mathbf{R}^{3}\right)\right)
$$


(ii) if $W_{n} \rightarrow W$ in $\mathcal{W}$, then

$$
\begin{aligned}
& \text { ess } \sup _{t \in(0, T)}\left\|W_{n}(t, \cdot)\right\|_{\mathbf{W}_{0}^{1, \infty}\left(\Omega ; \mathbf{R}^{3}\right)} \leq c, \\
& W_{n} \rightarrow W \quad \text { weakly }(*) \operatorname{in} \mathbf{L}^{\infty}\left(0, T ; \mathbf{W}^{1, \infty}(\Omega)\right) .
\end{aligned}
$$

Let

$$
\omega \in \mathcal{O} \mapsto\left\{\left[\rho_{0}(\cdot, \omega),(\rho \mathbf{u})_{0}(\cdot, \omega), \mathbf{H}_{0}(\cdot, \omega)\right], W\right\} \in \mathcal{I D} \times \mathcal{W}
$$

be a random variable.

Then there exists a random variable $\rho(\cdot, \omega), \mathbf{u}(\cdot, \omega)$, and $\mathbf{H}(\cdot, \omega)$,

$$
\begin{aligned}
& \rho(\cdot, \omega) \in \mathbf{C}\left([0, T] ; \mathbf{L}^{1}(\Omega)\right), \\
& \mathbf{u}(\cdot, \omega) \in \mathbf{L}^{2}\left(0, T ; \mathbf{W}_{0}^{1,2}(\Omega)\right), \\
& \mathbf{H}(\cdot, \omega) \in \mathbf{L}^{2}\left(0, T ; \mathbf{W}_{0}^{1,2}(\Omega)\right),
\end{aligned}
$$

satisfying the compressible magnetohydrodynamic equations (1.5)-(1.8) for a.a. $\omega \in \mathcal{O}$.

A simple example of $P(\rho)$ satisfying condition (1.10) is $P(\rho)=\frac{P_{\infty}}{p} \rho^{p}+a \rho$, which is not physical example. Here $p>\frac{3}{2}, P_{\infty}$, and $a$ are positive constants. Comparing with the existence of weak solution to the MHD equations without any stochastic effect (see e.g. [16]), the main difference of the weak solution obtained in this paper is the use of a random variable $(\rho(\cdot, \omega), \mathbf{u}(\cdot, \omega), \mathbf{H}(\cdot, \omega))$, for a.a. $\omega \in \mathcal{O}$.

This paper is organized as follows. In Section 2, we first recall the standard definition of weak solutions to the MHD system and give a stability results for the low regularity of the driving force, then we exploit it and show the existence of weak solutions for system (1.5)-(1.8) with a fixed (irregular) force. Finally, in the last section, we give the proof of Theorem 1.1.

\section{Existence of solutions for problems with irregular forces}

In this section, we give the existence of solutions for problems with irregular forces. Firstly, we prove the weak sequential stability result, which is at the heart of the proof of the main result. The stability means that a sequence of weak solutions to (1.5)-(1.8), with precompact data $\rho_{0},(\rho \mathbf{u})_{0}, \mathbf{H}_{0}$, and $W$, admits a subsequence that converges weakly to another solution of the same problem. This proof is based on the work of [12,16-18]. Before giving the stability result, we rewrite the weak formulation of the problem with dropping the parameter $\omega$ and fixing a function $W$.

- Equation of continuity:

$$
\begin{aligned}
& \int_{0}^{T} \int_{\Omega}(\rho+b(\rho)) \partial_{t} \varphi+(\rho+b(\rho)) \mathbf{u} \cdot \nabla_{x} \varphi d x d t \\
& \quad=\int_{0}^{\tau} \int_{\Omega}\left(\left(b^{\prime}(\rho) \rho-b(\rho)\right) \operatorname{div}_{x} \mathbf{u}\right) \varphi d x d t-\int_{\Omega}\left(\rho_{0}+b\left(\rho_{0}\right)\right) \varphi(0, \cdot) d x
\end{aligned}
$$

for any test function $\varphi \in \mathbf{C}_{c}^{\infty}([0, T) \times \bar{\Omega})$, any $b \in \mathbf{C}_{c}^{\infty}[0, \infty)$. 
- The momentum equation:

$$
\begin{aligned}
\int_{0}^{T} & \int_{\Omega}\left(\rho(\mathbf{u}-W) \cdot \partial_{t} \varphi+\rho \mathbf{u} \otimes \mathbf{u}: \nabla_{x} \varphi+P(\rho) \mathbf{d i v}_{x} \varphi\right) d x d t \\
= & \int_{0}^{\tau} \int_{\Omega}\left(\frac{1}{2}|\mathbf{H}|^{2} \cdot \nabla_{x} \varphi+(\mathbf{H} \cdot \nabla) \mathbf{H} \cdot \varphi\right) d x d t \\
& +\int_{0}^{\tau} \int_{\Omega}\left(v \nabla_{x} \mathbf{u} \cdot \nabla_{x} \varphi+(v+\lambda)\left(\operatorname{div}_{x} \mathbf{u}\right) \cdot \nabla_{x} \varphi\right. \\
& \left.+\rho(t, x, \omega) \mathbf{u} \cdot \nabla_{x}(W \varphi)\right) d x d t-\int_{\Omega}(\rho \mathbf{u})_{0} \cdot \varphi(0, \cdot) d x
\end{aligned}
$$

for all $\varphi \in \mathbf{C}_{c}^{\infty}\left([0, T) \times \Omega ; \mathbf{R}^{3}\right)$.

- The induction equation of electromagnetism:

$$
\begin{aligned}
\int_{0}^{T} & \int_{\Omega}\left(\mathbf{H} \cdot \partial_{t} \varphi+v\left(\nabla_{x} \times \mathbf{H}, \nabla_{x} \times \varphi\right)\right) d x d t \\
\quad & \int_{0}^{T} \int_{\Omega}\left(\mathbf{u} \times \mathbf{H}, \nabla_{x} \times \varphi\right) d x d t-\int_{\Omega} \mathbf{H}_{0} \cdot \varphi(0, \cdot) d x
\end{aligned}
$$

for all $\varphi \in \mathbf{C}_{c}^{\infty}\left([0, T) \times \Omega ; \mathbf{R}^{3}\right)$.

- The energy inequality:

$$
\begin{aligned}
-\int_{0}^{T} & \int_{\Omega}\left(\frac{1}{2} \rho|\mathbf{u}-W|^{2}+P(\rho)+\frac{1}{2}|\mathbf{H}|^{2}\right) d x d t \\
& +\int_{0}^{T} \int_{\Omega}\left(\nu\left|\nabla_{x} \mathbf{u}\right|^{2}+(\nu+\lambda)\left|\mathbf{d i v}_{x} \mathbf{u}\right|^{2}+v\left|\nabla_{x} \times \mathbf{H}\right|^{2}\right) d x \\
\leq & \varphi(0) \int_{\Omega}\left(\frac{1}{2} \frac{\left|(\rho \mathbf{u})_{0}\right|^{2}}{\rho_{0}}+P\left(\rho_{0}\right)+\frac{1}{2}\left|\mathbf{H}_{0}\right|^{2}\right) d x \\
& -\int_{0}^{\tau} \int_{\Omega}\left(\rho \mathbf{u} \otimes \mathbf{u}: \nabla_{x} W+P(\rho) \mathbf{d i v}_{x} W-\frac{1}{2} \rho \mathbf{u} \cdot \nabla_{x}|W|^{2}\right) d x d t \\
& -\int_{0}^{\tau} \int_{\Omega}\left(v \nabla_{x} \mathbf{u} \cdot \nabla_{x} W+(\nu+\lambda) \mathbf{d i v}_{x} \mathbf{u} \cdot \nabla_{x} W\right) d x d t \\
& +\int_{0}^{\tau} \int_{\Omega}\left(\frac{1}{2}|\mathbf{H}|^{2} \cdot \nabla_{x} W+(\mathbf{H} \cdot \nabla) \mathbf{H} \cdot W\right) d x d t \\
& +\int_{0}^{\tau} \int_{\Omega}\left(\mathbf{u} \times \mathbf{H}, \nabla_{x} \times W\right) d x d t
\end{aligned}
$$

for any $\varphi \in \mathbf{C}_{c}^{\infty}[0, T), \varphi \geq 0$.

Proposition 2.1 Let $\Omega \subset \mathbf{R}^{3}$ be a bounded Lipschitz domain. Assume that the pressure P satisfies (1.10). Let $\left\{W_{n}\right\}_{n=1}^{\infty}$ be a sequence of functions which satisfies

$$
\operatorname{ess} \sup _{t \in(0, T)}\left\|W_{n}(t, \cdot)\right\|_{\mathbf{W}_{0}^{1, \infty}\left(\Omega ; \mathbf{R}^{3}\right)} \leq C_{W}, \quad W_{n} \rightarrow W \text { in } \mathbf{L}^{1}\left(0, T ; \mathbf{W}_{0}^{1,1}\left(\Omega ; \mathbf{R}^{3}\right)\right)
$$


Let $\left\{\rho_{n}, \mathbf{u}_{n}, \mathbf{H}_{n}\right\}$ be a sequence of weak solutions of the MHD system driven by $W_{n}$ in $(0, T) \times$ $\Omega$, with the initial data $\rho_{0, n},(\rho \mathbf{H})_{0, n}, \mathbf{H}_{0, n}$ such that

$$
\begin{aligned}
& \rho_{0, n} \geq 0, \quad \int_{\Omega} \rho_{0, n} d x=M>0, \quad\left\|\rho_{0, n}\right\|_{\mathbf{L}^{p}(\Omega)} \leq E_{0}, \quad \int_{\Omega} \frac{\left|(\rho \mathbf{u})_{0, n}\right|^{2}}{\rho_{0, n}} d x \leq E_{0}, \\
& \rho_{0, n} \rightarrow \rho_{0} \quad \text { in } \mathbf{L}^{p}(\Omega), \quad(\rho \mathbf{u})_{0, n} \rightarrow(\rho \mathbf{u})_{0} \quad \text { weakly in } \mathbf{L}^{1}(\Omega), \\
& \int_{\Omega} \frac{\left|(\rho \mathbf{u})_{0, n}\right|^{2}}{\rho_{0, n}} d x \rightarrow \int_{\Omega} \frac{\left|(\rho \mathbf{u})_{0}\right|^{2}}{\rho_{0}} d x .
\end{aligned}
$$

Then, at least for suitable subsequences,

$$
\begin{aligned}
& \rho_{n} \rightarrow \rho \quad \text { in } \mathbf{C}_{\text {weak }}\left(0, T ; \mathbf{L}^{p}(\Omega)\right) \text { and in } \mathbf{L}^{1}((0, T) \times \Omega), \\
& \mathbf{u}_{n} \rightarrow \mathbf{u} \quad \text { weakly in } \mathbf{L}^{2}\left(0, T ; \mathbf{W}_{0}^{1,2}\left(\Omega, \mathbf{R}^{3}\right)\right), \\
& \mathbf{H}_{n} \rightarrow \mathbf{H} \quad \text { weakly in } \mathbf{L}^{2}\left(0, T ; \mathbf{W}_{0}^{1,2}\left(\Omega, \mathbf{R}^{3}\right)\right), \\
& \rho_{n}\left(\mathbf{u}_{n}-W_{n}\right) \rightarrow \rho(\mathbf{u}-W) \quad \text { in } \mathbf{C}_{\text {weak }}\left([0, T] ; \mathbf{L}^{\frac{2 p}{p+1}}\left(\Omega ; \mathbf{R}^{3}\right)\right),
\end{aligned}
$$

where $\rho, \mathbf{u}$, and $\mathbf{H}$ is a weak solution of the same problem driven by the force $W$, and having the initial data $\rho_{0},(\rho \mathbf{u})_{0}$, and $\mathbf{H}_{0}$.

Proof We first derive some uniform bounds estimates, which are exactly the same as the a priori bounded estimates available for the compressible MHD, $c f$. [16], and the stochastic compressible Navier-Stokes expression (see [12]). Applying the standard Gronwall inequality to the energy inequality (2.4), we get

$$
\begin{aligned}
& \text { ess } \sup _{t \in(0, T)}\left\|\rho_{n}(t, \cdot)\right\|_{\mathbf{L}^{p}(\Omega)} \leq c\left(M, C_{w}, E_{0}, T\right), \\
& \text { ess } \sup _{t \in(0, T)}\left\|\sqrt{\rho_{n}} \mathbf{u}_{n}(t, \cdot)\right\|_{\mathbf{L}^{2}\left(\Omega ; \mathbf{R}^{3}\right)} \leq c\left(M, C_{w}, E_{0}, T\right), \\
& \text { ess } \sup _{t \in(0, T)}\left\|\mathbf{H}_{n}(t, \cdot)\right\|_{\mathbf{L}^{2}\left(\Omega ; \mathbf{R}^{3}\right)} \leq c\left(M, C_{w}, E_{0}, T\right),
\end{aligned}
$$

where $M$ is the total mass, which is a constant. Moreover, one has

$$
\left\|\rho_{n}(t, \cdot)\right\|_{\mathbf{L}^{1}(\Omega)}=M \quad \forall t \in[0, T] .
$$

Moreover, by assumption (2.5),

$$
\text { ess } \sup _{t \in(0, T)} \int_{\Omega} \rho\left|W_{n}\right|^{2} d x \leq c\left(M, C_{w}\right) .
$$

We use Korn's inequality,

$$
\begin{aligned}
& \int_{0}^{T}\left\|\mathbf{u}_{n}(t, \cdot)\right\|_{\mathbf{w}_{0}^{1,2}\left(\Omega ; \mathbf{R}^{3}\right)}^{2} d t \leq c\left(M, C_{w}, E_{0}, T\right), \\
& \int_{0}^{T}\left\|\mathbf{H}_{n}(t, \cdot)\right\|_{\mathbf{w}_{0}^{1,2}\left(\Omega ; \mathbf{R}^{3}\right)}^{2} d t \leq c\left(M, C_{w}, E_{0}, T\right) .
\end{aligned}
$$


Consequently, there exists a suitable subsequence such that

$$
\begin{aligned}
& \rho_{n} \rightarrow \rho \quad \text { in } \mathbf{C}_{\text {weak }}\left(0, T ; \mathbf{L}^{p}(\Omega)\right) \\
& \mathbf{u}_{n} \rightarrow \mathbf{u} \quad \text { weakly in } \mathbf{L}^{2}\left(0, T ; \mathbf{W}_{0}^{1,2}\left(\Omega, \mathbf{R}^{3}\right)\right), \\
& \mathbf{H}_{n} \rightarrow \mathbf{H} \quad \text { weakly in } \mathbf{L}^{2}\left(0, T ; \mathbf{W}_{0}^{1,2}\left(\Omega, \mathbf{R}^{3}\right)\right), \\
& \rho_{n} \mathbf{u}_{n} \rightarrow \rho \mathbf{u} \quad \text { weakly }(*) \text { in } \mathbf{L}^{\infty}\left(0, T ; \mathbf{L}^{\frac{2 p}{p+1}}\left(\Omega, \mathbf{R}^{3}\right)\right) .
\end{aligned}
$$

By the momentum equation (2.2), we derive

$$
\rho_{n}\left(\mathbf{u}_{n}-W_{n}\right) \rightarrow \rho(\mathbf{u}-W) \quad \text { in } \mathbf{C}_{\text {weak }}\left([0, T] ; \mathbf{L}^{\frac{2 p}{p+1}}\left(\Omega ; \mathbf{R}^{3}\right)\right),
$$

which combining with (2.5) and (2.12) gives

$$
\begin{aligned}
& \left(\rho_{n} \mathbf{u}_{n}\right)(t, \cdot) \rightarrow(\rho \mathbf{u})(t, \cdot) \quad \text { weakly in } \mathbf{L}^{\frac{2 p}{p+1}}\left(\Omega ; \mathbf{R}^{3}\right) \text { for a.a. } t \in(0, T), \\
& \left(\rho_{n} \mathbf{u}_{n}\right)(t, \cdot) \rightarrow(\rho \mathbf{u})(t, \cdot) \quad \text { in } \mathbf{L}^{q}\left(0, T ; \mathbf{W}^{-1,2}\left(\Omega ; \mathbf{R}^{3}\right)\right), \forall 1 \leq q<\infty
\end{aligned}
$$

By (2.6)-(2.7), (2.10), and (2.13), we have

$$
\rho_{n} \mathbf{u}_{n} \otimes \mathbf{u}_{n} \rightarrow \rho \mathbf{u} \otimes \mathbf{u} \quad \text { weakly in } \mathbf{L}^{2}\left(0, T ; \mathbf{L}^{\frac{6 p}{4 p+3}}\left(\Omega ; \mathbf{R}^{3 \times 3}\right)\right) .
$$

Using (2.10)-(2.11), we get

$$
\begin{aligned}
& \left(\nabla \mathbf{H}_{n} \times \mathbf{H}_{n}\right) \times \mathbf{H}_{n} \rightarrow(\nabla \mathbf{H} \times \mathbf{H}) \times \mathbf{H} \quad \text { in } \mathcal{D}((0, T) \times \Omega), \\
& \nabla \times\left(\mathbf{u}_{n} \times \mathbf{H}_{n}\right) \rightarrow \nabla \times(\mathbf{u} \times \mathbf{H}) \quad \text { in } \mathcal{D}((0, T) \times \Omega),
\end{aligned}
$$

where we use $\mathcal{D}^{\prime}$ to denote the sense of distributions.

Next, we derive the pressure estimate and the pointwise convergence of the densities. We denote the Bogovskii operator as $\mathcal{B} \approx \mathbf{d i v}^{-1}$, and we choose the test function

$$
\varphi=\psi(t) \mathcal{B}\left[b\left(\rho_{n}\right)-\frac{1}{|\Omega|} \int_{\Omega} b\left(\rho_{n}\right) d x\right], \quad \psi \in \mathbf{C}_{c}^{\infty}(0, T), b(\rho) \approx \rho^{\kappa} .
$$

Following [12] and [16] step by step, we deduce that

$$
\int_{0}^{T} \int_{\Omega} P\left(\rho_{n}\right) \rho_{n}^{\beta} \leq c\left(M, C_{w}, E_{0}, T\right)
$$

for a certain positive constant $\beta$ which depends on $M, C_{w}$, and $E_{0}$.

To derive the pointwise convergence of the densities

$$
\rho_{n} \rightarrow \rho \quad \text { a.a. in }(0, T) \times \Omega,
$$

we take the following test function:

$$
\varphi=\psi \eta(x) \nabla_{x} \triangle^{-1}[\xi \overline{b(\rho)}]
$$


in the limit equation

$$
\begin{aligned}
& \int_{0}^{T} \int_{\Omega}\left(\rho(\mathbf{u}-W) \cdot \partial_{t} \varphi+\rho \mathbf{u} \otimes \mathbf{u}: \nabla_{x} \varphi+\overline{P(\rho)} \mathbf{d i v}_{x} \varphi\right) d x d t \\
&= \int_{0}^{\tau} \int_{\Omega}\left(\frac{1}{2}|\mathbf{H}|^{2} \cdot \nabla_{x} \varphi+(\mathbf{H} \cdot \nabla) \mathbf{H} \cdot \varphi\right) d x d t \\
& \quad+\int_{0}^{\tau} \int_{\Omega}\left(v \nabla_{x} \mathbf{u} \cdot \nabla_{x} \varphi+(v+\lambda)\left(\operatorname{div}_{x} \mathbf{u}\right) \cdot \nabla_{x} \varphi\right. \\
&\left.\quad+\rho \mathbf{u} \cdot \nabla_{x}(W \varphi)\right) d x d t-\int_{\Omega}(\rho \mathbf{u})_{0} \cdot \varphi(0, \cdot) d x,
\end{aligned}
$$

where $\overline{b(\rho)}$ or $\overline{P(\rho)}$ denotes a weak limit of the compositions $\overline{b\left(\rho_{n}\right)}$ or $\overline{P\left(\rho_{n}\right)}$.

Using the same arguments as in $[12,19]$, we have

$$
\begin{gathered}
\lim _{n \rightarrow \infty} \int_{0}^{T} \int_{\mathbf{R}^{3}} \psi \mathbf{u}_{n} \cdot\left(\nabla_{x} \Delta^{-1} \nabla_{x}\left[\xi b\left(\rho_{n}\right)\right] \cdot \eta \rho_{n} \mathbf{u}_{n}-\xi b\left(\rho_{n}\right) \nabla_{x} \triangle^{-1} \nabla_{x}\left[\eta \rho_{n} \mathbf{u}_{n}\right]\right) d x d t \\
=\int_{0}^{T} \int_{\mathbf{R}^{3}} \psi \mathbf{u} \cdot\left(\nabla_{x} \Delta^{-1} \nabla_{x}[\xi b(\rho)] \cdot \eta \rho \mathbf{u}-\xi \overline{b(\rho)} \nabla_{x} \Delta^{-1} \nabla_{x}[\eta \rho \mathbf{u}]\right) d x d t .
\end{gathered}
$$

By (2.5)-(2.9), we derive

$$
\rho_{n} W_{n} \rightarrow \rho W \quad \text { in } \mathbf{L}^{2}\left(0, T ; \mathbf{W}^{-1, q^{\prime}}\left(\Omega ; \mathbf{R}^{3}\right)\right)
$$

which implies that

$$
\begin{aligned}
\lim _{n \rightarrow \infty} & \int_{0}^{T} \int_{\mathbf{R}^{3}}\left[\psi \rho_{n} \mathbf{u}_{n} \cdot \nabla_{x}\left(\eta W_{n} \cdot \nabla_{x} \Delta^{-1}\left[\xi b\left(\rho_{n}\right)\right]\right)\right. \\
& \left.+\rho_{n} W_{n} \cdot \partial_{t}\left(\psi \eta \nabla_{x} \Delta^{-1}\left[\xi b\left(\rho_{n}\right)\right]\right)\right] d x d t \\
= & \lim _{n \rightarrow \infty} \int_{0}^{T} \int_{\mathbf{R}^{3}}\left[\psi \rho \mathbf{u} \cdot \nabla_{x}\left(\eta W \cdot \nabla_{x} \Delta^{-1}[\xi \overline{b(\rho)}]\right)\right. \\
& \left.+\rho W \cdot \partial_{t}\left(\psi \eta \nabla_{x} \triangle^{-1}[\xi \overline{b(\rho)}]\right)\right] d x d t
\end{aligned}
$$

By (2.19)-(2.20), we obtain

$$
\begin{gathered}
\lim _{n \rightarrow \infty} \int_{0}^{T} \int_{\mathbf{R}^{3}} \xi \eta\left(\xi P\left(\rho_{n}\right) b\left(\rho_{n}\right)-\left(v \nabla_{x} \mathbf{u}_{n}+(v+\lambda)\left(\operatorname{div}_{x} \mathbf{u}_{n}\right)\right) \cdot \nabla_{x} \triangle^{-1} \nabla_{x}\left[\xi b\left(\rho_{n}\right)\right]\right) d x d t \\
\quad=\int_{0}^{T} \int_{\mathbf{R}^{3}} \xi \eta\left(\xi \overline{P(\rho) b(\rho)}-\left(v \nabla_{x} \mathbf{u}+(v+\lambda)\left(\operatorname{div}_{x} \mathbf{u}\right)\right) \cdot \nabla_{x} \triangle^{-1} \nabla_{x}[\xi \overline{b(\rho)}]\right) d x d t .
\end{gathered}
$$

In particular, we have the so-called weak continuity of the effective viscous flux

$$
\overline{P(\rho) b(\rho)}-\left(\frac{4}{3} v+\lambda\right) \overline{b(\rho)}\left(\operatorname{div}_{x} \mathbf{u}\right)=\overline{P(\rho) b(\rho)}-\left(\frac{4}{3} v+\lambda\right) \overline{b(\rho)} \mathbf{d i v}_{x} \mathbf{u},
$$

which first was established in [20] and recovered in [12] for stochastic compressible Navier-Stokes system. 
Now the rest of deriving (2.21) is by the same arguments as in [12,19]. Moreover, for any $1 \leq q \leq p$, we can obtain

$$
\rho_{n} \rightarrow \rho \quad \text { in } \mathbf{C}\left([0, T] ; \mathbf{L}^{q}(\Omega)\right) .
$$

Assume that $W$ is regular and satisfies

$$
W \in \mathbf{C}^{1}\left([0, T] ; W^{1, \infty}\left(\Omega ; \mathbf{R}^{3}\right)\right), \quad W(0, \cdot)=0 .
$$

As shown in $[12,16]$, by a small modification of the proof for the compressible MHD system and using Proposition 2.1, we obtain the following result.

Proposition 2.2 Let $\Omega \subset \mathbf{R}^{3}$ be a bounded Lipschitz domain. Assume that the pressure $P$ satisfies (1.10),

$$
W \in \mathbf{L}_{\text {weak }}^{\infty}\left(0, T ; W_{0}^{1, \infty}\left(\Omega ; \mathbf{R}^{3}\right)\right)
$$

and

$$
\begin{aligned}
& \rho_{0} \in \mathbf{L}^{p}(\Omega), \quad \rho_{0} \geq 0, \quad \int_{\Omega} \rho_{0}=M>0, \\
& (\rho \mathbf{u})_{0} \in \mathbf{L}^{1}\left(\Omega, \mathbf{R}^{3}\right), \quad \int_{\Omega} \frac{\left|\rho \mathbf{u}_{0}\right|^{2}}{\rho_{0}} d x<\infty, \quad \mathbf{H}_{0} \in \mathbf{L}^{2}(\Omega) .
\end{aligned}
$$

Then the compressible MHD system admits a weak solution in $(0, T) \times \Omega$ in the sense specified in (2.1)-(2.4).

Remark 2.1 We can use a sequence of approximation smooth function $W_{n}$ for any regular $W$ to obtain solutions for the problem driven by any regular $W$. The symbol $\mathbf{L}_{\text {weak }}^{\infty}$ denotes the space of weakly measurable functions.

\section{Proof of Theorem 1.1}

Assume that

$$
\omega \in \mathcal{O} \mapsto W(\cdot, \omega) \in \mathcal{W}
$$

is a random variable, and the mapping

$$
\omega \in \mathcal{O} \mapsto\left[\rho_{0}(\cdot, \omega),(\rho \mathbf{u})_{0}(\cdot, \omega), \mathbf{H}_{0}(\cdot, \omega)\right] \in \mathcal{I D}
$$

is a random variable on $\mathcal{O}=\{\mathcal{O}, \mathcal{B}, \mu\}$, with a regular probability measure $\mu$. Then for any $\varepsilon>0$, there exists a compact set $\mathcal{K}_{\varepsilon \subset \mathcal{O}}$ such that

$$
\mu\left(\mathcal{O} \backslash \mathcal{K}_{\varepsilon}\right)<\varepsilon,
$$

and the mapping

$$
\omega \mapsto\left[\rho_{0}(\cdot, \omega),(\rho \mathbf{u})_{0}(\cdot, \omega), \mathbf{H}_{0}(\cdot, \omega)\right]
$$

restricted to $\mathcal{K}_{\varepsilon}$ is continuous. 
Consider a multi-valued mapping

$$
\begin{aligned}
\mathcal{L}:\left\{\left[\rho_{0},(\rho \mathbf{u})_{0}, \mathbf{H}_{0}\right], W\right\} & \in \mathcal{I D} \times \mathcal{W} \mapsto[\rho, \mathbf{u}, \mathbf{H}] \\
& \in \mathbf{C}\left([0, T] ; \mathbf{L}^{1}(\Omega)\right) \times \mathbf{L}^{2}\left(0, T ; \mathbf{W}_{0}^{1,2}\left(\Omega, \mathbf{R}^{3}\right)\right) .
\end{aligned}
$$

The existence of $\mathcal{L}$ is guaranteed by Proposition 2.2. Proposition 2.1 implies that the value of $\mathcal{L}$ are non-empty closed subsets of the Banach space $\mathbf{C}\left([0, T] ; \mathbf{L}^{1}(\Omega)\right) \times \mathbf{L}^{2}\left(0, T ; \mathbf{W}_{0}^{1,2}(\Omega\right.$; $\left.\mathbf{R}^{3}\right)$ ), and $\mathcal{L}$ has a closed graph.

Finally, using the abstract result of Theorem 3.1 and Lemma 3.1 in [21], we conclude that there exists a weak solution of the MHD driven by the stochastic external force $W$ in the sense of (1.5)-(1.8) such that the mapping

$$
\omega \mapsto[\rho(\cdot, \omega), \mathbf{u}(\cdot, \omega), \mathbf{H}(\cdot, \omega)] \in \mathbf{C}\left([0, T] ; \mathbf{L}^{1}(\Omega)\right) \times \mathbf{L}^{2}\left(0, T ; \mathbf{W}_{0}^{1,2}\left(\Omega ; \mathbf{R}^{3}\right)\right)
$$

is a random variable, for $\omega \in \mathcal{O}$. This completes the proof of Theorem 1.1.

\section{Competing interests}

The authors declare that they have no competing interests.

\section{Authors' contributions}

All authors contributed equally to the writing of this paper. All authors read and approved the final manuscript.

\section{Author details}

'School of Mathematics and Information Science, Henan Polytechnic University, Jiaozuo, 454150, P.R. China. ${ }^{2}$ School of Mathematics and Information Sciences, North China University of Water Resources and Electric Power, Zhengzhou, 450011, P.R. China.

\section{Acknowledgements}

The authors express their sincere thanks to the anonymous referees for the comments.

Received: 27 September 2014 Accepted: 17 April 2015 Published online: 01 May 2015

\section{References}

1. Kulikovskiy, AG, Lifshitz, EM: Magnetohydrodynamics. Addison-Wesley, Reading (1965)

2. Laudau, LD, Lifshitz, EM: Electrodynamics of Continuous Media, 2nd edn. Pergamon, New York (1984)

3. Polovin, RV, Demutskii, VP: Fundamentals of Magnetohydrodynamics. Consultants Bureau, New York (1990)

4. Bensoussan, A: Stochastic Navier-Stokes equations. Acta Appl. Math. 38, 268-304 (1995)

5. Flandoli, F: An Introduction to 3D Stochastic Fluid Dynamics. CIME Lecture Notes (2005)

6. Mattingly, J: On recent progress for the stochastic Navier-Stokes equations with non-Lipschitz random forcing. Rev. Math. Phys. 22, 669-697 (2010)

7. Flandoli, F, Gatarek, D: Martingale and stationary solutions for stochastic Navier-Stokes equations. Probab. Theory Relat. Fields 102, 367-391 (1995)

8. Flandoli, F, Romito, M: Markov selections for 3D stochastic Navier-Stokes equation. Probab. Theory Relat. Fields 140 407-458 (2008)

9. Goldys, B, Röckner, M, Zhang, XC: Martingale solutions and Markov selections for stochastic partial differential equations. Stoch. Process. Appl. 119, 1725-1764 (2009)

10. Tornatore, E, Fujita Yashima, $\mathrm{H}$ : One-dimensional equations of a barotropic viscous gas with a not very regular perturbation. Ann. Univ. Ferrara, Sez. VII 40, 137-168 (1994)

11. Tornatore, E, Fujita Yashima, H: One-dimensional stochastic equations for a viscous barotropic gas. Ric. Mat. 46(2), 255-283 (1997)

12. Feireisl, E, Maslowski, B, Novotny, A: Compressible fluid flows driven by stochastic forcing. J. Differ. Equ. 254 1342-1358 (2013)

13. Sritharan, SS, Sundar, P: The stochastic magneto-hydrodynamic system. Infin. Dimens. Anal. Quantum Probab. Relat. Top. 2, 241-265 (1999)

14. Sunda, P: Stochastic magnetohydrodynamic system perturbed by general noise. Commun. Stoch. Anal. 4, 253-269 (2010)

15. Diperna, R-J, Lions, P-L: Ordinary differential equations, transport theory and Sobolev spaces. Invent. Math. 98 511-547 (1989)

16. Hu, X, Wang, D: Global existence and large time behavior of solutions to the three dimensional equations of compressible magnetohydrodynamic flows. Arch. Ration. Mech. Anal. 197, 203-238 (2010)

17. Coifman, R, Meyer, Y: On commutators of singular integrals and bilinear singular integrals. Trans. Am. Math. Soc. 212 , 315-331 (1975) 
18. Yan, WP: Existence of weak solutions to the three-dimensional density-dependent generalized incompressible magnetohydrodynamic flows. Discrete Contin. Dyn. Syst., Ser. A 35(3), 1359-1385 (2015)

19. Feireisl, E, Novotny, A, Petzeltova, $\mathrm{H}$ : On the existence of globally defined weak solutions to the Navier-Stokes equations of compressible isentropic fluids. J. Math. Fluid Mech. 3, 358-392 (2001)

20. Lions, P-L: Mathematical Topics in Fluid Dynamics. Volume 2: Compressible Models. Oxford University Press, Oxford (1998)

21. Bensoussan, A, Temam, R: Équations stochastiques du type Navier-Stokes. J. Funct. Anal. 13, 195-222 (1973)

Submit your manuscript to a SpringerOpen ${ }^{\circ}$ journal and benefit from:

- Convenient online submission

Rigorous peer review

- Immediate publication on acceptance

- Open access: articles freely available online

- High visibility within the field

- Retaining the copyright to your article 